\title{
Papers
}

\section{Outcome of expectant management of spontaneous first trimester miscarriage: observational study}

\author{
Ciro Luise, Karen Jermy, Caroline May, Gillian Costello, William P Collins, Thomas H Bourne
}

\begin{abstract}
Objectives To evaluate the uptake and outcome of expectant management of spontaneous first trimester miscarriage in an early pregnancy assessment unit. Participants 1096 consecutive patients with a diagnosis of spontaneous first trimester miscarriage. Methods Each miscarriage was classified as complete, incomplete, missed, or anembryonic on the basis of ultrasonography. Women who needed treatment were given the choice of expectant management or surgical evacuation of retained products of conception under general anaesthesia. Women undergoing expectant management were checked a few days after transvaginal bleeding had stopped, or they were monitored at weekly intervals for four weeks. Main outcome measures A complete miscarriage (absence of transvaginal bleeding and endometrial thickness $<15 \mathrm{~mm}$ ), the number of women completing their miscarriage within each week of management, and complications (excessive pain or transvaginal bleeding necessitating hospital admission or clinical evidence of infection).

Results Two patients with molar pregnancies were excluded, and $37 \%$ of the remainder (408/1094) were classified as having had a complete miscarriage. $70 \%$ (478/686) of women with retained products of conception chose expectant management; of these, 27 $(6 \%)$ were lost to follow up. A successful outcome without surgical intervention was seen in $81 \%$ of cases $(367 / 451)$. The rate of spontaneous completion was $91 \%(201 / 221)$ for those cases classified as incomplete miscarriage, $76 \%(105 / 138)$ for missed miscarriage, and $66 \%(61 / 92)$ for anembryonic pregnancy. $70 \%$ of women completed their miscarriage within 14 days of classification (84\% for incomplete miscarriage and 52\% for missed miscarriage and anembryonic pregnancy).

Conclusions Most women with retained products of conception chose expectant management. Ultrasonography can be used to advise patients on the likelihood that their miscarriage will complete spontaneously within a given time.
\end{abstract}

\section{Introduction}

About $15 \%$ of women with a clinically recognised pregnancy will miscarry spontaneously during the first trimester. ${ }^{1}$ Current management often involves the surgical evacuation of retained products of conception even though serious complications-for example, infection, uterine perforation, or bowel damage-may arise. $^{2}$ Early pregnancy assessment units open to patients without the need for referral and the use of transvaginal ultrasonography have enabled the presence and stage of early pregnancy failure to be determined from direct images. Preliminary data arising from these developments have shown that expectant management with serial monitoring may be used to identify those patients who will not require surgery. ${ }^{3-5}$ More studies are required on the acceptability of this management strategy and on the relation between the information derived from ultrasound scans and the clinical outcome. We report an observational study designed to assess the uptake and effectiveness of expectant management for different clinical presentations of spontaneous miscarriage in consecutive unselected patients attending an early pregnancy assessment unit.

\section{Patients, methods, and results}

A total of 1096 consecutive patients with a suspected first trimester miscarriage were observed. Each miscarriage was classified as complete, incomplete, missed, or anembryonic according to guidelines from the Royal College of Obstetricians and Gynaecologists (table 1). Each participant was counselled about the implications of her condition. Women classified as having a complete miscarriage were asked to report any further worrying signs or symptoms. Those with retained products of conception were offered the choice of expectant management or the surgical removal of retained products of conception under general anaesthesia. Women undergoing expectant management had the right to change their management at any time. Their progress was checked a few days after transvaginal bleeding had stopped or through weekly monitoring for four weeks. Women who were asymptomatic, but had not completed the miscarriage were advised to have surgery. The main outcome measures were a complete miscarriage (the absence of transvaginal bleeding and an endometrial thickness $<15 \mathrm{~mm}$ ), the number of women who had completed their miscarriage by the end of each week from the day of classification, and complications (excessive pain or transvaginal bleeding necessitating hospital admission or clinical evidence of infection). Patients requesting or

\author{
Early Pregnancy, \\ Gynaecological \\ Ultrasound and \\ Minimal Access \\ Surgery Unit, St \\ George's Hospital, \\ London SW17 0RE \\ Ciro Luise \\ clinical research fellow \\ Karen Jermy \\ clinical research fellow \\ Caroline May \\ medical student \\ Gillian Costello \\ midwife \\ Thomas H Bourne \\ consultant \\ Academic \\ Department of \\ Obstetrics and \\ Gynaecology, Guy's, \\ King's and St \\ Thomas's School of \\ Medicine, King's \\ College Hospital, \\ London SE5 9PJ \\ William P Collins \\ emeritus professor \\ Correspondence to: \\ T Bourne \\ tbourne@ \\ gynae-scanning.com
} BMJ 2002;324:873-5 
Table 1 Classification of spontaneous miscarriage at diagnosis in an observational study

\begin{tabular}{lccl} 
Classification & $\begin{array}{c}\text { Transvaginal bleeding } \\
\text { (No/yes) }\end{array}$ & $\begin{array}{c}\text { Endometrial thickness* } \\
(\mathbf{m m})\end{array}$ & Products of conception \\
\hline Complete miscarriage & No & $<15$ & None \\
\hline Incomplete miscarriage & No/yes & Any & $\begin{array}{l}\text { Heterogeneous tissues (with or without a gestational sac) distorting the } \\
\text { endometrial midline echo }\end{array}$ \\
\hline Missed miscarriage & No/yes & Any & $\begin{array}{l}\text { Fetal pole (crown-rump length }>6 \mathrm{~mm}, \text { no heart beat) or if crown-rump length } \\
\leqslant 6 \mathrm{~mm} \text { with no change on rescan } 7 \text { days later }\end{array}$ \\
\hline Anembryonic pregnancy & No/yes & Any & $\begin{array}{l}\text { Gestational sac (diameter }>20 \mathrm{~mm}, \text { no embryonic pole or yolk sac) or if } \\
\text { diameter } \leqslant 20 \mathrm{~mm} \text { with no change } 7 \text { days later }\end{array}$ \\
\hline
\end{tabular}

${ }^{\star}$ Endometrial thickness = maximum distance from one myometria-endometrial interface to another.

requiring surgical treatment were placed on a list for elective day surgery, and they were treated in the standard way by experienced operators.

Two patients with molar pregnancies were excluded. Of the remaining 1094 patients, 408 (37\%) were classified as having completed a miscarriage. Of the remaining 686 patients, $478(70 \%)$ chose expectant management; of these, $12(3 \%)$ asked for more than four weeks before deciding whether to undergo surgery, and they were given a further three weeks to decide (three completed their miscarriage after 32, 36, and 46 days). After $27(6 \%)$ patients were lost to follow up, data from 451 patients were available for analysis (table 2). A successful spontaneous outcome with no serious complications was observed in $367(81 \%)$ of cases. None of the 408 patients initially classified as having had a complete miscarriage reported an ectopic pregnancy or any other serious complication requiring intervention. The overall rate of spontaneous completion for cases classified as incomplete miscarriage was 201/221 (91\%); the value for missed miscarriage was $105 / 138(76 \%)$ and for anembryonic pregnancies $61 / 92(66 \%)$ (table 2). Overall, 52\% of incomplete miscarriages had resolved spontaneously by day 7 of management and $84 \%$ by day 14 . The corresponding values for missed miscarriages and anembryonic pregnancies were $28 \%$ by day 7 and $56 \%$ by day 14 . Complications arose in 11/1094 (1\%) patients; of these, 5/208 (2\%) patients had undergone immediate surgical removal of the products of conception and 6/451 (1\%) were undergoing expectant management. One patient who had an incomplete miscarriage and was managed expectantly required emergency surgery and a blood transfusion.

\section{Discussion}

In our observational study, most patients with retained products of conception chose expectant management; they were probably influenced by the knowledge that they could rapidly receive surgical treatment if required. We believe that the success rate of expectant management was dependent on the extent and quality of patient counselling, particularly when transvaginal blood loss was great (which may be associated with a higher rate of complete miscarriage). The lower rate of complications in women who chose expectant management is reassuring, although this finding depends on many factors, which will vary with the setting.

Our data show that the classified results of transvaginal ultrasonography can be used to advise patients of the likelihood that their miscarriage will complete spontaneously within given periods of time from the day of classification. It is also reassuring that the ultrasound criteria for a complete miscarriage in the absence of transvaginal bleeding seem to give valid results. We are aware, however, that some patients may have experienced problems that were not reported to us, and they may have received additional advice or treatment elsewhere.

Further studies are needed to obtain more data on the usefulness of transvaginal ultrasonography to define an outcome measure for a complete miscarriage and to validate and improve endpoints for the classification of first trimester miscarriages. The current odds in favour of a successful outcome for patients with an incomplete miscarriage, a missed miscarriage, and an anembryonic pregnancy are about 9:1, 3:1, and 2:1, respectively.

Patients can now be encouraged to persevere with expectant management, because of the high completion rate by day 14 from classification. The implementation of these findings will reduce the number of women undergoing surgery.

This article was initially submitted to the $B M J$ as a full length paper. After peer review, the authors changed the format to a short report. A perusal of the proofs revealed that the text did not adequately describe the follow up procedure. Subsequently, the implications of this led to a reassessment of the aims of the study and changes to the statistical analysis. The original (unpublished) manuscripts were based on the consideration that 1094 patients underwent expectant management and were monitored in a similar way for any unsatisfactory developments or symptoms. The re-evaluation of follow up data for each patient showed that those classified as having a completed miscarriage at the time of the first visit had not, in practice, been monitored so closely (or completely) as those with an incomplete miscarriage, a missed miscarriage, or an anembryonic pregnancy. Accordingly, in this published report, those patients with a complete miscarriage according to criteria derived from ultrasound at the first visit are considered

Table 2 Types of miscarriage and outcomes in patients who chose expectant management. Values are numbers (percentages)

\begin{tabular}{|c|c|c|c|c|}
\hline \multirow[b]{2}{*}{ Group classification at diagnosis } & \multirow[b]{2}{*}{ Patients } & \multicolumn{2}{|c|}{ Complete miscarriage } & \multirow{2}{*}{$\begin{array}{l}\text { Successful outcome by } \\
\text { day } 46\end{array}$} \\
\hline & & By day 7 & By day 14 & \\
\hline Incomplete miscarriage & $221(49)$ & $117(53)$ & $185(84)$ & 201(91) \\
\hline Missed miscarriage & $138(31)$ & $41(30)$ & $81(59)$ & $105(76)$ \\
\hline Anembryonic pregnancy & $92(20)$ & $23(25)$ & $48(52)$ & $61(66)$ \\
\hline Total & $451(100)$ & $181(40)$ & $314(70)$ & $367(81)$ \\
\hline
\end{tabular}




\section{What is known already on this topic}

The management of spontaneous first trimester miscarriage is often based on digital assessment of the cervical os, ultrasonography, and the surgical evacuation of retained products of conception

Expectant management, in early pregnancy assessment units, may be useful for some women and would reduce the overall number of women undergoing surgery

\section{What this study adds}

Most women who miscarry in the first trimester choose expectant management and about $81 \%$ of these complete their miscarriage without intervention

Ultrasonography provides a useful assessment of whether a miscarriage will complete without intervention within a given time

separately from patients in the other three groups. The same basic data and outcome measures were used in the reanalysis.
The short report was expanded to include more information, and it was resubmitted and accepted as a short paper.

Contributors: CL initiated the research, participated in the protocol design, coordinated patient recruitment, performed transvaginal scans, provided counselling, collected and analysed data, and contributed to writing the paper. $\mathrm{KJ}$ participated in the protocol design, performed transvaginal scans, and provided counselling. CM participated in the collection of data and analysis of results. GC performed transvaginal scans and provided counselling. WC interpreted, discussed, and presented the data and contributed to writing the paper. TB coordinated the preparation of the protocol, discussed core ideas, analysed data, and contributed to writing the paper. THB is the guarantor.

Funding: None.

Competing interests: None declared.

Steer C, Campbell S, Davies M, Mason B, Collins WP. Spontaneous abortion rates after natural and assisted conception. BMJ 1989;299:1317-8.

MacKenzie J, Bibby J. Critical assessment of dilatation and curettage in 1029 women. Lancet 1978;ii:566-8.

3 Nielsen S, Hahlin M, Platz-Christiansen JJ. Randomised study comparing expectant management with medical management for first trimester miscarriages. Br J Obstet Gynaecol 1999;106:804-7.

4 Jurkovic D, Ross JA, Nicolaides KH. Expectant management of missed miscarriage. Br J Obstet Gynaecol 1998;105:670-1.

5 Schwarzler P, Holden D, Nielsen S, Hahlin M, Sladkevicius P, Bourne TH The conservative management of first trimester miscarriages and the use of colour Doppler sonography for patient selection. Hum Reprod 1999:14:1341-5.

6 RCR/RCOG Working Party. Early pregnancy assessment. London: RCOG Press, 1996.

(Accepted 5 April 2001)

\title{
Treatment of imported malaria in an ambulatory setting: prospective study
}

\author{
Valérie D’Acremont, Pierre Landry, Roger Darioli, Dieter Stuerchler, Alain Pécoud, Blaise Genton
}

Many specialists in tropical medicine consider that patients with imported malaria, at least those with Plasmodium falciparum malaria, should be admitted to hospital, as complications can develop quickly. ${ }^{1}$ In Switzerland, patients with malaria who lack signs of severe disease are treated as outpatients, because empirical observations of patients with imported malaria show that death is usually due to a delay in diagnosis rather than complications during treatment. ${ }^{2}$ We conducted a prospective study in the outpatient clinic of a university hospital to assess the safety of treating imported malaria in an ambulatory setting.

\section{Participants, methods, and results}

We conducted our study from January 1990 to July 2000. At study entry we used predefined clinical and laboratory criteria (table) to determine if patients with malaria required admission to hospital. If no criteria were met, ambulatory treatment was considered appropriate. Patients received the first dose of drugs under supervision and were kept under surveillance for one hour before being sent home with instructions. Follow up was at the attending doctor's discretion: clinical and parasitological assessments were performed daily until symptoms resolved and one blood slide was clear of parasites.

Overall, 165 (17\%) of 958 patients with fever were positive for parasites; $113(69 \%)$ had $P$ falciparum. Sev-
Predefined clinical and laboratory criteria for admission of patients with malaria to hospital and number of patients primarily admitted to hospital with the condition

\begin{tabular}{|c|c|}
\hline Criteria & $\begin{array}{c}\text { No }(\%) \text { of patients } \\
(\mathrm{n}=36)\end{array}$ \\
\hline \multicolumn{2}{|l|}{ Clinical } \\
\hline Poor general condition & $23(64)$ \\
\hline Repeated vomiting & $9(25)$ \\
\hline Temperature $>40^{\circ} \mathrm{C}$ & $9(25)$ \\
\hline Hypotension & $4(11)$ \\
\hline Any neurological problem & $5(14)$ \\
\hline Any respiratory problem & 0 \\
\hline Any bleeding sign & 0 \\
\hline Jaundice & $5(14)$ \\
\hline Failure with previous antimalarial treatment & 0 \\
\hline Poor compliance & 0 \\
\hline Alone at home & 0 \\
\hline Pregnancy & 0 \\
\hline \multicolumn{2}{|l|}{ Laboratory } \\
\hline Parasitaemia $>2 \%$ & $8(22)$ \\
\hline Platelets <20 g/l & $6(17)$ \\
\hline Haemoglobin <80 g/dl & 0 \\
\hline Creatinine $>250 \mu \mathrm{mol} / \mathrm{ml}$ & 0 \\
\hline Glycaemia $<3.9 \mathrm{mmol} / \mathrm{l}$ & 0 \\
\hline
\end{tabular}

enty one $(43 \%)$ of the 165 were first generation immigrants and none was white; and 135 (82\%) had travelled to Africa. Median age was 33.7 (range 16-76) years, and median time from onset of symptoms to consultation was four days. Seventy seven (47\%) 